PROCEEDINGS OF THE AMERICAN MATHEMATICAL SOCIETY

Volume 124, Number 11, November 1996

\title{
IRREDUCIBLE POSITIVE LINEAR MAPS ON OPERATOR ALGEBRAS
}

\author{
DOUGLAS R. FARENICK
}

(Communicated by Palle E. T. Jorgensen)

\begin{abstract}
Motivated by the classical results of G. Frobenius and O. Perron on the spectral theory of square matrices with nonnegative real entries, D. Evans and R. Høegh-Krohn have studied the spectra of positive linear maps on general (noncommutative) matrix algebras. The notion of irreducibility for positive maps is required for the Frobenius theory of positive maps. In the present article, irreducible positive linear maps on von Neumann algebras are explicitly constructed, and a criterion for the irreducibility of decomposable positive maps on full matrix algebras is given.
\end{abstract}

Let $A$ and $A^{+}$denote a $C^{*}$-algebra and its cone of positive elements. A linear map $\varphi: A \rightarrow A$ is positive if it leaves the cone $A^{+}$invariant; that is, $\varphi(x) \in A^{+}$for every $x \in A^{+}$. If $A$ is an $n$-dimensional commutative $C^{*}$-algebra, then a positive map $\varphi$ on $A$ has a representation as an $n \times n$ matrix with nonnegative real entries. Therefore, the classical matrix theoretic results (see [7, Ch.8]) of O. Perron and G. Frobenius on matrices with nonnegative entries can be viewed as an important case of a more general theory that deals with positive maps on operator algebras. This is the viewpoint taken by D. Evans and R. Høegh-Krohn [6], S. Albeverio and R. Høegh-Krohn [2], and U. Groh [8], [9] in their works on the spectra of positive maps on operator algebras. In both the classical theory and its various generalisations [12], and in the one put forward in [6], [2], [8], and [9], a positive map that is "strictly" positive, or that is "irreducible", will possess certain interesting spectral properties. Although the spectral theory of such maps has been studied, the issue of how one is to determine whether a given positive linear map on an operator algebra is strictly positive or irreducible (or neither) has received much less attention. For matrices with nonnegative entries a simple and readily verifiable criterion exists, dating back to Frobenius: $\varphi$ is strictly positive if and only if each entry of $\varphi$ is positive, and $\varphi$ is irreducible if and only if the directed graph of $\varphi$ is strongly connected. However for positive maps on noncommutative operator algebras, it is somewhat more difficult to make this determination. Indeed this difficulty appears to be hampered even further by the fact that there is no tractable structure theory for positive maps.

Received by the editors May 2, 1995.

1991 Mathematics Subject Classification. Primary 46L05.

Key words and phrases. Positive linear maps, irreducibilty, completely positive maps.

This work is supported in part by a grant from The Natural Sciences and Engineering Research Council of Canada.

(C)1996 American Mathematical Society 
The purpose of this article is twofold. First of all we aim to develop a "usable" criterion for the irreducibility of certain positive maps on operator algebras; this is done in order to facilitate the construction of concrete irreducible positive maps to accompany the more theoretical works [2], [6], [8] on spectral theory. The second point to be made here is that the notion of irreducibility for positive maps on operator algebras is combinatorial, just as it is for nonnegative matrices; this fact is of interest because, in general, cone-preserving maps are not usually explicitly described by combinatorial data.

In what follows the term operator algebra is to mean a von Neumann algebra $A$, with identity 1 , of operators acting on some complex Hilbert space $H$. For the definition and study of irreducible positive linear maps on $C^{*}$-algebras, see the papers of Groh [8], [9].

Definition. A face $F$ of the cone $A^{+}$is a trivial face if $F=A^{+}$or $F=\{0\}$. A positive linear map $\varphi$ on $A$ is said to be strictly positive if $\varphi(x)$ is bounded below for every nonzero $x \in A^{+}$, and is called irreducible if there are no nontrivial weaklyclosed faces $F$ of $A^{+}$that are invariant under $\varphi$. We say that $\varphi$ is reducible if $\varphi$ is not irreducible.

The definition of reducibility, as stated above, is not in fact how Evans and Høegh-Krohn define this term in that they use, instead, invariant hereditary subalgebras induced by projections. The definition given here follows the tradition [12] of developing Frobenius theory in a manner whereby reducibility is viewed in terms of invariant faces of $A^{+}$. Regardless, these two viewpoints are equivalent.

Proposition 1. The following statements are equivalent for a positive map $\varphi$ on a von Neumann algebra A.

(1) There is a nontrivial weakly-closed face of $A^{+}$that is invariant under $\varphi$.

(2) There is a nontrivial projection $p \in A$ and a real number $\lambda>0$ such that $\varphi(p) \leq \lambda p$.

(3) There is a nontrivial projection $p \in A$ such that the hereditary $C^{*}$-subalgebra $p A p$ is invariant under $\varphi$.

Proof. To prove that (1) implies (2), we assume without loss of generality that $\|\varphi\| \leq 1$, and that $F$ is a nontrivial weakly-closed face of $A^{+}$that is invariant under $\varphi$, and that if $F$ is minimal, then $F \neq\{\lambda 1\}$ for some real $\lambda \geq 0$. Let $F_{1}=\left\{x \in A^{+}: x \in F,\|x\| \leq 1\right\}$, which is a weakly-closed face of the positive part of the unit ball of $A$. By [1,2.2], there exist unique projections $p_{1}, p_{2} \in A$ such that $F_{1}=\left\{x \in A^{+}: p_{1} \leq x \leq p_{2}\right\}$. Because $\varphi$ is a contraction and $F$ is $\varphi$-invariant, $F_{1}$ is invariant under $\varphi$. Now as $F_{1}$ is a nontrivial face, at least one of $p_{1}, p_{2}$ is a nontrivial projection. If $p_{2} \neq 1$, then $\varphi\left(p_{2}\right) \in F_{1}$ implies that $\varphi\left(p_{2}\right) \leq p_{2}$, which yields the desired conclusion. If $p_{1} \neq 1$, then $p_{2} \neq 0$ and $p_{2} \neq 1$ (because of the restrictions on $F$ ), and $\varphi(1) \leq 1$, as $\varphi(1) \in F_{1}$. Hence, $1-p_{1} \geq 1-\varphi\left(p_{1}\right) \geq \varphi(1)-\varphi\left(p_{1}\right)=\varphi\left(1-p_{1}\right)$, which is the desired conclusion.

Assume now that (2) holds; so there is a nontrivial projection $p \in A$ and a scalar $\lambda>0$ such that $\varphi(p) \leq \lambda p$. If $a \in A^{+}$, then $p a p \leq\|a\| p$ and so, by the positivity of $\varphi, \varphi($ pap $) \leq\|a\| \varphi(p) \leq \lambda\|a\| p$. Hence $\varphi($ pap $) \in(p A p)^{+}$. As every $x \in A$ is a linear combination of elements of the form pap, for certain $a \in A^{+}$, it follows that $\varphi$ leaves $p A p$ invariant.

Lastly, suppose that the hereditary $C^{*}$-subalgebra $p A p$ is $\varphi$-invariant, where $p$ is a nontrivial projection. Let $F=\left\{x \in A^{+}: x \leq \mu p\right.$ for some real $\left.\mu>0\right\}$, a 
weakly-closed nontrivial face of $A^{+}$. If $x \in F$, then $x \leq \mu p$ for some real $\mu>0$ implies that $x \in p A p$ and so $x=p a p$ for some $a \in A$. Hence $\varphi(x) \leq \lambda p$ for every $\lambda>\|a\|$, and so $\varphi(x) \in F$.

Using Proposition 1, one can deduce some straightforward necessary conditions that must be satisfied in order for a positive linear map $\varphi$ on $A$ to be irreducible.

Corollary 1. If $\varphi$ is an irreducible positive linear map on a von Neumann algebra $A$, then

(1) $\operatorname{ker} \varphi \cap A^{+}=\{0\}$;

(2) $\varphi+\psi$ is irreducible for every positive map $\psi$ on $A$;

(3) $\psi$ is irreducible if $\psi$ is positive and $\varphi \leq \psi$;

(4) $\varphi(x)$ has dense range in $H$ for every $x \in A^{+}$with dense range.

Proof. Statements (2) and (3) are evident. To prove (1), suppose that $x \in \operatorname{ker} \varphi \cap$ $A^{+}$, and that $x \neq 0$. If $x=\lambda 1$, then for every $a \in A^{+}, a \leq\|a\| 1$ implies that $\varphi(a) \leq \lambda\|a\| \varphi(1)=0$ and so $\varphi$ is identically zero, which an irreducible map cannot be. Thus we may assume $x$ is nonzero and has at least two spectral values; this means that if $E$ is the spectral resolution of the identity for $x$, then there is a real number $0<\delta<\|x\|$ such that $p=E(\delta, \infty)$ is a nontrivial projection. From

$$
x=\int_{0}^{\infty} \lambda d E(\lambda) \geq \int_{\delta}^{\infty} \lambda d E(\lambda) \geq \delta \int_{\delta}^{\infty} d E(\lambda)=\delta p,
$$

we have that $\delta \varphi(p) \leq \varphi(x)=0 \leq p$, which contradicts the irreducibility of $\varphi$. Hence it must be that $x=0$.

For statement (4), consider $\varphi(1)$ to begin with. If $\varphi(1)$ does not have dense range, then the range projection $R[\varphi(1)]$ of $\varphi(1)$ is nontrivial. From $R[\varphi(1)] \leq 1$ we have that $\varphi(R[\varphi(1)]) \leq \varphi(1) \leq\|\varphi(1)\| R[\varphi(1)]$ and so $\varphi$ is reducible, which contradicts our hypothesis that $\varphi$ is irreducible. Therefore the range of $\varphi(1)$ must be dense in $H$. Now suppose that $x$ is a positive invertible element of $A$. Then there is a real number $\delta>0$ such that $0<\delta 1 \leq x$; hence, $\delta \varphi(1) \leq \varphi(x)$. Now if $\varphi(x)$ fails to have dense range, then there exists a nonzero vector $\xi \in H$ that is annihilated by $\varphi(x)$. The inequality $\delta \varphi(1) \leq \varphi(x)$ implies, therefore, that $\varphi(1) \xi=0$, which is in contradiction to the fact that $\varphi(1)$ has dense range. Hence, it must be that the range of $\varphi(x)$ is dense in $H$.

The criterion for irreducibility given by Evans and Høegh-Krohn is a direct parallel to one used by linear algebraists, although it is difficult to use for practical purposes; nevertheless, the criterion is important in that it shows an exact relationship between irreducibility and strict positivity.

An irreducibility criterion. Suppose that $\varphi$ is a positive linear map on a von Neumann algebra $A$ of operators acting on an $n$-dimensional Hilbert space, and that $\tau$ is a faithful, normalised trace on A. The following statements are equivalent.

(1) $\varphi$ is irreducible.

(2) There is a positive integer $k<n$ such that $(I+\varphi)^{k}$ is strictly positive.

(3) For each nonzero pair of $x, y \in A^{+}$with $\tau\left(y^{*} x\right)=0$ there is a positive integer $k<n$ such that $\tau\left(y^{*} \varphi^{k}(x)\right)>0$.

A few of the main results concerning spectral theory, in the finite-dimensional case, are summarised in the following theorem. See [2] and [8] for analogous results in infinite dimensions. 
Theorem 1 ([6]). Suppose that $\varphi$ is a positive linear map on a finite-dimensional von Neumann algebra $A$, and let $r$ denote the spectral radius of $\varphi$.

(1) There is a nonzero $z \in A^{+}$such that $\varphi(z)=r z$.

(2) If $\varphi$ is irreducible and if $y \in A^{+}$is an eigenvector of $\varphi$ corresponding to some eigenvalue $\alpha$ of $\varphi$, then $\alpha=r$ and $y$ is a positive scalar multiple of $z$.

(3) If $\varphi$ is irreducible, unital, and satisfies the Schwarz inequality for positive linear maps, then

(a) $r=1$ and $\operatorname{ker}(\varphi-1)=\mathbb{C} 1$;

(b) every eigenvalue $\zeta \in \sigma(\varphi) \cap \mathbb{T}$ is simple and the eigenspace $\operatorname{ker}(\varphi-\zeta 1)$ is spanned by a unitary $u_{\zeta} \in A$ which satisfies $\varphi\left(u_{\zeta} x\right)=\zeta u_{\zeta} \varphi(x)$, for every $x \in A$;

(c) $\sigma(\varphi) \cap \mathbb{T}$ is a cyclic subgroup $\Gamma$ of $\mathbb{T}$, and the eigenvectors $u_{\zeta}$ determined by $\zeta \in \Gamma$ (as in (b) above) form a cyclic group, isomorphic to $\Gamma$ under the map $\zeta \mapsto u_{\zeta}$. The map $\varphi$ is said to be primitive if $\Gamma$ is trivial; if $\Gamma$ is nontrivial, then $\varphi$ is said to be imprimitive of order $|\Gamma|$.

(4) If $\varphi$ is strictly positive, unital, and satisfies the Schwarz inequality, then $r=1$ is the only eigenvalue of unit modulus.

In order to produce nontrivial examples of irreducible positive maps, and in some cases to characterise all irreducible maps within a specific class, Theorem 2 below will be our main tool. The description in Theorem 2 is combinatorial, as it depends on invariant subspace lattices. This is in keeping with the flavour of Frobenius's matrix-theoretic notion of irreducibility, which is typically viewed as a property concerning directed graphs. Recall that a directed graph $\mathfrak{G}$ on $n$ vertices is said to be strongly connected if for each pair of distinct vertices $v_{i}$ and $v_{j}$ there is a directed path in $\mathfrak{G}$ connecting $v_{i}$ with $v_{j}$. The directed graph of a matrix $T \in M_{n}(\mathbb{C})$ is the directed graph which contains an edge directed from vertex $v_{i}$ to vertex $v_{j}$ if and only if the $(i, j)$-entry of the matrix $T$ is nonzero; a matrix $T$ is said to be irreducible if and only if its directed graph is strongly connected. It is useful to note here that matrix-theoretic irreducibility is also a property concerning the invariant subspace lattice: namely, a matrix $T$ is irreducible in the sense of Frobenius if and only if $T$ has no nontrivial invariant subspace that is spanned by a subset of the standard orthonormal basis vectors $\xi_{1}, \ldots, \xi_{n}$ of $\mathbb{C}^{n}$. For a more explicit link between Frobenius's notion of irreducibility and irreducible positive linear maps on matrix algebras, refer to Example 2.

A collection $S$ of (closed) subspaces of a Hilbert space $H$ will be called trivial if $S$ contains only $\{0\}$ and $H$. Given an operator $a \in B(H)$, Lat- $a$ will denote the invariant subspace lattice of $a$.

Theorem 2. Suppose that a positive linear map $\varphi$ on a von Neumann algebra $A$ has the form $\varphi(x)=\sum_{j} a_{j}^{*} x a_{j}$, for some operators $a_{j} \in B(H)$ for which $\sum_{j} a_{j}^{*} x a_{j}$

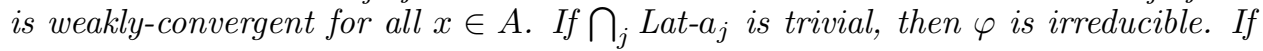
$A=M_{n}(\mathbb{C})$, then $\bigcap_{j}$ Lat- $a_{j}$ is necessarily trivial when $\varphi$ is irreducible.

Proof. Suppose that the operators $a_{j} \in B(H)$ are such that $\bigcap_{j}$ Lat- $a_{j}$ is trivial, and assume that $\varphi(p) \leq \lambda p$ for some real $\lambda \geq 0$ and projection $p \in A$. If $\xi \in \operatorname{ker} p$, then

$$
0 \leq(\varphi(p) \xi, \xi)=\sum_{j}\left(p a_{j} \xi, a_{j} \xi\right) \leq \lambda(p \xi, \xi)=0
$$


implies that $\left(p a_{j} \xi, a_{j} \xi\right)=0$ for each $j$, which is equivalent to $p a_{j} \xi=0$ for each $j$. Thus ker $p \in \bigcap_{j}$ Lat- $a_{j}$ and so $\operatorname{ker} p$ is either $H$ or $\{0\}$. Hence, $\varphi$ has no nontrivial invariant projections.

Suppose, conversely, that $\varphi$ is an irreducible positive map on $M_{n}(\mathbb{C})$ of the form $\varphi(x)=\sum_{j} a_{j}^{*} x a_{j}$. Assume $K \in \bigcap_{j}$ Lat- $a_{j}$ is a nontrivial subspace; we will prove that this assumption contradicts the irreducibility of $\varphi$. Let $q$ be the projection onto $K^{\perp}$. Because $K$ is invariant under each $a_{j}$, with respect to the decomposition $\mathbb{C}^{n}=K \oplus K^{\perp}, q$ and each $a_{j}$ is an operator matrix:

$$
q=\left(\begin{array}{ll}
0 & 0 \\
0 & 1
\end{array}\right) \text { and } a_{j}=\left(\begin{array}{cc}
z_{j} & s_{j} \\
0 & g_{j}
\end{array}\right)
$$

Hence

$$
\varphi(q)=\sum_{j}\left(\begin{array}{cc}
0 & 0 \\
0 & g_{j}^{*} g_{j}
\end{array}\right) \leq\left\|\sum_{j} g_{j}^{*} g_{j}\right\|\left(\begin{array}{cc}
0 & 0 \\
0 & 1
\end{array}\right)=\left\|\sum_{j} g_{j}^{*} g_{j}\right\| q,
$$

which implies that $\varphi$ is reducible, a contradiction.

Example 1. If $a \in A$ is an irreducible operator in $B(H)$, and if $\psi$ is an arbitrary positive linear map on $A$, then the map $\varphi$ on $A$ defined by $\varphi(x)=a^{*} x a+a x a^{*}+\psi(x)$, for every $x \in A$, is an irreducible positive linear map.

Proof. Because $a$ is irreducible, $a$ and $a^{*}$ have no common nontrivial invariant subspaces and therefore the map $x \mapsto a^{*} x a+a x a^{*}$ is irreducible. By (2) of Corollary 1 , the addition of $\psi$ to an irreducible map produces another irreducible map.

Example 1 gives a very simple and explicit method for constructing irreducible positive linear maps on $A$ and, in particular, on $B(H)$. The remainder of the paper treats positive maps on the matrix algebra $M_{n}(\mathbb{C})$, for it is here where the Evans/Høegh-Krohn spectral theory is developed and where the connections with Frobenius's notion of irreducibility are the strongest. At the end of the article, one further construction for general operator algebras is discussed.

To fully link the notion of irreducibility for positive maps with combinatorics, it is necessary to assume these positive maps have some sort of structure, namely that they are decompsable. Recall that a positive linear map is decomposable [14] if it is a sum of completely positive and copositive linear maps. If $A$ is the full matrix algebra $M_{n}(\mathbb{C})$, then from the theorem [4] of M.-D. Choi, every decomposable map $\varphi$ is of the form $\varphi=\psi+\varrho$, where $\psi$ is completely positive and of the form $\psi(x)=\sum_{j=1}^{k} a_{j}^{*} x a_{j}$, for some canonical choice of linearly independent matrices $a_{1}, \ldots, a_{k} \in M_{n}(\mathbb{C})$, and $\varrho$ is copositive, of the form $\varrho(x)=\sum_{i=1}^{m} b_{i}^{*} x^{t} b_{i}$, for some $b_{1}, \ldots, b_{m}$. As decomposable maps on $M_{n}(\mathbb{C})$ have a defined structure, Theorem 2 can be modified slightly to describe the irreducibility of these maps in terms of the canonical matricial coefficients $a_{j}, b_{i}$ that arise.

Irreducible Decomposable Maps. If $\varphi$ is a positive linear map on $M_{n}(\mathbb{C})$ of the form $\varphi(x)=\sum_{j} a_{j}^{*} x a_{j}+\sum_{i} b_{i}^{*} x^{t} b_{i}$, for some matrices $a_{1}, \ldots, a_{k}, b_{1}, \ldots, b_{m} \in$ $M_{n}(\mathbb{C})$, then $\varphi$ is irreducible if and only if $\left(\bigcap_{j}\right.$ Lat- $\left._{j}\right) \cap\left(\bigcap_{i}\right.$ Lat- $\left._{i}\right)$ is trivial. (It is implicit that the cardinality of the set $\left\{a_{1}, \ldots, a_{k}, b_{1}, \ldots, b_{m}\right\}$ is at least 2.)

Proof. If $\varphi$ is irreducible, then the latter part of the proof of Theorem 2 applies equally well here to yield the conclusion that $\left(\bigcap_{j}\right.$ Lat- $\left.a_{j}\right) \cap\left(\bigcap_{i}\right.$ Lat- $\left.b_{i}\right)$ is trivial. 
Conversely, assume that $\left(\bigcap_{j}\right.$ Lat- $\left.a_{j}\right) \cap\left(\bigcap_{i}\right.$ Lat- $\left.b_{i}\right)$ is trivial, and that $\varphi(p) \leq \lambda p$, for some projection $p$ and nonzero positive real number $\lambda$. An application of the arguments used in Theorem 2 shows that if $\xi \in \operatorname{ker} p$, then $a_{j} \xi, \overline{b_{i} \xi} \in \operatorname{ker} p$ for all $i, j$; the vector $\bar{\eta}$ and the matrix $\bar{a}$ denote the vector and matrix obtained from $\eta \in \mathbb{C}^{n}$ and $a \in M_{n}(\mathbb{C})$ by taking the complex conjugate of each entry. It remains, then, to prove that $p b_{i} \xi=0$ for each $i$. Because $p^{*}=p, p\left(\overline{b_{i} \xi}\right)=0$ implies that $p^{*}\left(\overline{b_{i} \xi}\right)=0$; so $\left(p+p^{*}\right) \overline{b_{i} \xi}=0$ and thus $\overline{p+p^{*}}\left(b_{i} \xi\right)=\overline{0}=0$. As each entry of $p+p^{*}$ is real, we have that $2 p\left(b_{i} \xi\right)=0$ and so $p\left(b_{i} \xi\right)=0$.

Corollary 2. The following positive linear maps on $M_{n}(\mathbb{C})$ are reducible.

(1) Every inner automorphism of $M_{n}(\mathbb{C})$.

(2) Every inner antiautomorphism of $M_{n}(\mathbb{C})$.

(3) Every positive map sending the cone $M_{n}(\mathbb{C})^{+}$onto itself.

(4) Every decomposable map $\varphi$ of the form $\varphi(x)=\sum_{j} a_{j}^{*} x a_{j}+\sum_{i} b_{i}^{*} x^{t} b_{i}$, where the matrices $a_{1}, \ldots, a_{k}, b_{1}, \ldots, b_{m}$ are commuting. In this case, if $\sigma$ is the joint spectrum of $a_{1}, \ldots, a_{k}, b_{1}, \ldots, b_{m}$, then the spectral radius of $\varphi$ is the maximum of the moduli of all inner products $(\mu, \nu)$ in $\mathbb{C}^{k+m}$, as $\mu$ and $\nu$ vary throughout the joint spectrum $\sigma$.

Proof. If $u$ is unitary, then the maps $x \mapsto u^{*} x u$ and $x \mapsto u^{*} x^{t} u$ are reducible; this proves (1) and (2). A positive map sending $M_{n}(\mathbb{C})^{+}$onto itself is decomposable and of the form $x \mapsto a^{*} x a$ or of the form $x \mapsto a^{*} x^{t} a$, for some $a \in M_{n}(\mathbb{C})$ [13]; such maps are, by the criterion, reducible. Finally for (4), commuting matrices have a common eigenvector, which spans a common 1-dimensional invariant subspace. The assertion concerning the spectral radius of $\varphi$ is a special case of a theorem in [11].

With the following example we have one case where the combinatorial information provided by Theorem 2 can be set in a graph-theoretic context. The standard matrix units of $M_{n}(\mathbb{C})$ are the $n^{2}$ matrices $e_{i j}$ that have $(i, j)$-entry equal to 1 and all other entries equal to 0 . Unital positive maps are sometimes called stochastic maps; if $\tau$ denotes a faithful normalised trace on $A$, then a positive linear map $\varphi$ on $A$ is said to be doubly stochastic if $\varphi$ is stochastic and trace-preserving. The doubly stochastic maps on an $n$-dimensional commutative algebra are precisely the doubly stochastic matrices, that is, the set of $n \times n$ matrices $S$ with nonnegative entries such that the sum in each row and each column of $S$ is 1 .

Example 2. Let $\mathfrak{E}$ be a nonempty subset of the set $\mathfrak{U}$ of standard matrix units for $M_{n}(\mathbb{C})$, and consider the positive linear map on $M_{n}(\mathbb{C})$ defined by $\varphi(x)=$ $\sum_{a \in \mathfrak{E}} a^{*} x a$, for all $x$.

(1) $\varphi$ is irreducible if and only if the matrix $E=\sum_{a \in \mathfrak{E}} a$ is irreducible in the sense of Frobenius.

(2) $\varphi$ is doubly stochastic if and only if the matrix $E=\sum_{a \in \mathfrak{E}} a$ is doubly stochastic.

(3) $\varphi$ is strictly positive if and only if $\mathfrak{E}=\mathfrak{U}$; equivalently, $\varphi$ is strictly positive if and only if, for each $x, \varphi(x)=\tau_{x} 1$, where $\tau_{x}$ is the (nonnormalised) trace of $x$.

Proof. Suppose that $\varphi$ is irreducible. Let $\mathfrak{S}_{n}$ denote the group of permutations on $n$ elements; for each $\sigma \in \mathfrak{S}_{n}$, let $p_{\sigma}$ be the unitary matrix that maps each standard orthonormal basis vector $\xi_{j}$ to $\xi_{\sigma(j)}$. Let $E=\sum_{a \in \mathfrak{E}} a$, a zero-one matrix. Assume 
that $E$ is reducible in the sense of Frobenius; then there is a $\sigma \in \mathfrak{S}_{n}$ such that $p_{\sigma}^{-1} E p_{\sigma}$ is a block matrix of the form $\left(\begin{array}{cc}E_{11} & E_{12} \\ 0 & E_{22}\end{array}\right)$, where $E_{11}$ is a $k \times k$ zero-one matrix with $k<n$. Thus, the subspace spanned by $\xi_{\sigma^{-1}(1)}, \ldots, \xi_{\sigma^{-1}(k)}$ is nontrivial and $E$-invariant. We can interpret $p_{\sigma}^{-1} E p_{\sigma}$ as a sum of matrix units as well: namely,

$$
p_{\sigma}^{-1} E p_{\sigma}=\sum_{e_{\mu \nu} \in \mathfrak{E}} e_{\sigma(\mu) \sigma(\nu)} .
$$

As $\operatorname{Span}\left\{\xi_{1}, \ldots, \xi_{k}\right\}$ is evidently invariant for every matrix unit $e_{\sigma(\mu) \sigma(\nu)}$, where $e_{\mu \nu} \in \mathfrak{E}$, then $\operatorname{Span}\left\{\xi_{\sigma^{-1}(1)}, \ldots, \xi_{\sigma^{-1}(k)}\right\}$ is invariant for every matrix unit $e_{\mu \nu} \in \mathfrak{E}$. But this contradicts the fact that, by Theorem 2, the matrix units $a \in \mathfrak{E}$ have no common nontrivial invariant subspaces; hence the matrix $E$ must be irreducible.

Assume, conversely, that $E=\sum_{a \in \mathfrak{E}} a$ is an irreducible nonnegative matrix in $M_{n}(\mathbb{R})$. Let $K \in \bigcap_{a \in \mathfrak{E}}$ Lat- $a$ be a nonzero subspace of minimal dimension. We shall prove that $K=\mathbb{C}^{n}$. Because $K$ is $a$-invariant for each $a \in \mathfrak{E}, K$ is invariant for (the irreducible matrix) $E$ as well. Each matrix unit $a \in \mathfrak{E}$ is of rank 1; hence, given a matrix unit $a$, either $a(K)=\{0\}$, or $a(K)=\operatorname{Span}\left\{\xi_{i}\right\}$ if $a=e_{i j}$ for some $j$. Therefore the subspace $L$ of $K$ generated by $\bigcup_{a \in \mathfrak{E}} a(K)$ is spanned by a subset of the standard orthonormal basis vectors of $\mathbb{C}^{n}$; moreover, $L$ is $a$-invariant for each $a \in \mathfrak{E}$ and so, by the minimality hypothesis, $L=K$. Hence $K$ is an $E$-invariant subspace that is spanned by some subset of the standard orthonormal basis of $\mathbb{C}^{n}$; by the irreducibility of $E$, this means that $K=\mathbb{C}^{n}$. By Theorem 2, the condition $\bigcap_{a \in \mathfrak{E}}$ Lat- $a=\left\{0, \mathbb{C}^{n}\right\}$ is equivalent to the irreducibility of $\varphi(\cdot)=\sum_{a \in \mathfrak{E}} a^{*}(\cdot) a$. This completes the proof of statement (1).

If $E=\sum_{a \in \mathfrak{E}} a$ is doubly stochastic, then because $E$ is a zero-one matrix, $E$ must be a permutation matrix: $E=p_{\sigma}$, for some $\sigma \in \mathfrak{S}_{n}$. Therefore, $\varphi$ is the map $\varphi(x)=\sum_{j} x_{j j} e_{\sigma(j) \sigma(j)}$, which is plainly doubly stochastic. Conversely, if $\varphi$ is doubly stochastic, then for each $j$ the matrix $\varphi\left(e_{j j}\right)$ is a matrix unit and has trace 1 ; as $\varphi(1)=1$, it follows that $\varphi$ is a permutation on the set of projections $e_{11}, \ldots, e_{n n}$, and so there is a permutation $\sigma \in \mathfrak{S}_{n}$ such that $\varphi\left(e_{j j}\right)=e_{\sigma(j) \sigma(j)}$, for all $j$. Fix $j$; if $e_{\mu \nu} \in \mathfrak{E}$, then $e_{\nu \mu} e_{j j} e_{\mu \nu}$ is either 0 or a matrix unit $e_{j \nu}$, and so $e_{\nu \mu} e_{j j} e_{\mu \nu}=e_{\sigma(j) \sigma(j)}$ if and only if $\mu=j$ and $\nu=\sigma(j)$. Thus, from

$$
\varphi\left(e_{j j}\right)=\sum_{e_{\mu \nu} \in \mathfrak{E}} e_{\nu \mu} e_{j j} e_{\mu \nu}=e_{\sigma(j) \sigma(j)}, \text { for every } j,
$$

we conclude that $\mathfrak{E}=\left\{e_{j \sigma(j)}: 1 \leq j \leq n\right\}$ and that $E=p_{\sigma}$, a doubly stochastic matrix.

We now establish statement (3). Assume to begin with that $\varphi$ is strictly positive. We will prove that $\mathfrak{E}$ must be the entire set $\mathfrak{U}$ of matrix units. This conclusion, in turn, is clearly equivalent to statement that $\varphi(x)$ has the form $x \mapsto \tau_{x} 1$, which implies that $\varphi$ is strictly positive. Suppose that $\mathfrak{E} \neq \mathfrak{U}$ and that $e_{\mu \nu} \in \mathfrak{U}$ but $e_{\mu \nu} \notin \mathfrak{E}$. If $a \in \mathfrak{E}$, then $e_{\mu \nu} a \neq 0$ only if $a=e_{\nu j}$ for some $j$; in this case, $a^{*} e_{\mu \nu} a=e_{j \nu} e_{\mu j}$, which is zero unless $\nu=\mu$. Thus, if $\mu \neq \nu$, then $\varphi$ sends $e_{\mu \nu}$ to zero. If $\nu=\mu$, then for every $a \in \mathfrak{E}, a^{*} e_{\mu \nu} a$ is either zero or a projection of the form $e_{j j}$, for some $j \neq \mu$. Thus

$$
\left(\varphi\left(e_{\mu \mu}\right) \xi_{\mu}, \xi_{\mu}\right)=\sum_{e_{j j} \in \mathfrak{E}}\left(e_{j j} \xi_{\mu}, \xi_{\mu}\right)=0 \quad(\text { as each } j \neq \mu),
$$


which implies that $\varphi\left(e_{\mu \mu}\right)$ is not invertible. Therefore, $\varphi$ cannot be a strictly positive map if $\mathfrak{E} \neq \mathfrak{U}$.

Observe that in Example 2 above, the range of $\varphi$ lies in the commutative subalgebra $D_{n}$ of diagonal matrices. The matrix $E$ is, therefore, intimately related to the map $\varphi$. Indeed the matrix representation of the restriction of $\varphi$ to $D_{n}$ is $E$; hence the Perron values of $\varphi$ and $E$ coincide.

The following proposition illustrates some of the properties held by strictly positive maps.

Proposition 2. The following statements are equivalent for a positive linear map $\varphi$ on $M_{n}(\mathbb{C})$.

(1) $\varphi$ is strictly positive.

(2) $\varphi(p)$ is invertible for every rank-1 projection $p$.

(3) $\varphi$ is in the interior of the cone of all positive linear maps on $M_{n}(\mathbb{C})$.

Proof. The equivalence of statements (2) and (3) is a theorem of S.-H. Kye [10]. Obviously statement (1) implies (2). Suppose, then, that statement (2) is true. Given any projection $p$, there is a rank-1 projection $q$ such that $q \leq p$. Thus, $\varphi(q) \leq \varphi(p)$ and $\varphi(q)$ invertible imply that $\varphi(p)$ is invertible. Now because the cone $M_{n}(\mathbb{C})^{+}$is generated by projections, it follows that $\varphi(x)$ is bounded below for each nonzero positive matrix $x$; that is, $\varphi$ is strictly positive.

An immediate consequence of this proposition is that every reducible positive linear map $\varphi$ on the $n \times n$ matrices lies on the boundary of the cone of all positive linear maps on $M_{n}(\mathbb{C})$. As the boundary of this cone will consist of both reducible and irreducible maps, the following example is of some interest.

Example 3. Every decomposable positive linear map $\varphi$ that generates an extremal ray in the cone of all decomposable positive linear maps on $M_{n}(\mathbb{C})$ is reducible.

Proof. Suppose that $\varphi$ that generates an extremal ray in the cone of decomposable positive linear maps on $M_{n}(\mathbb{C})$. By definition, $\varphi$ is a sum $\varphi=\psi+\varrho$, where $\psi$ is completely positive and $\varrho$ is copositive. From $\psi \leq \varphi$ and the fact that $\varphi$ generates an extremal ray, there is a real number $\mu \in[0,1]$ such that $\psi=\mu \varphi$; hence $(1-\mu) \psi=\mu \varrho$. Because $\psi$ is completely positive and $\varrho$ is not, $\mu$ must be either 0 or 1 . If $\mu=0$, then $\varphi$ is completely positive and generates an extremal ray in the cone of completely positive maps on $M_{n}(\mathbb{C})$; by [3, Cor1.4.3], $\varphi$ must therefore be a compression of an irreducible representation of $M_{n}(\mathbb{C})$. However as all irreducible representations of $M_{n}(\mathbb{C})$ are unitarily equivalent to the identity representation, $\varphi$ must have the form $\varphi(x)=a^{*} x a$, for all $x$, for some matrix $a$. By Theorem 2, such a map is reducible. Similar arguments show that in the case where $\mu=1$, there is a matrix $a$ such that $\varphi$ will have the form $\varphi(x)=a^{*} x^{t} a$, for all $x$, which is a reducible map by the criterion for irreducibility.

Is it possible to remove the condition that $\varphi$ be decomposable from the hypothesis of (3) above? In this direction it is noteworthy that the Choi-Lam example [5] of a nondecomposable map $\varphi$ on $M_{3}(\mathbb{C})$ generating an extremal ray in the cone of positive linear maps on $M_{3}(\mathbb{C})$ is reducible. The map is

$$
\varphi(x)=\frac{1}{2}\left(\begin{array}{ccc}
x_{11}+x_{33} & -x_{12} & -x_{13} \\
-x_{21} & x_{22}+x_{11} & -x_{23} \\
-x_{31} & -x_{32} & x_{33}+x_{22}
\end{array}\right)
$$


and the invariant hereditary subalgebra is $p M_{3}(\mathbb{C}) p$, where

$$
p=\frac{1}{3}\left(\begin{array}{lll}
1 & 1 & 1 \\
1 & 1 & 1 \\
1 & 1 & 1
\end{array}\right)
$$

Thus far the focus has been on factors such as $B(H)$ or $M_{n}(\mathbb{C})$. From finite direct sums of factors one can construct irreducible maps from certain maps between the factors. One simply follows matrix theory, and the construction is sketched here.

First of all, however, we say that a positive linear map $\varphi$ is dense-range positive if $\varphi(x)$ has dense range, for every nonzero $x \in A^{+}$. By definition, a strictly positive map is dense-range positive, and from the arguments used in Corollary 1, one sees that a dense-range positive map is irreducible. More generally, if $(I+\varphi)^{k}$ is dense-range positive, for some positive integer $k$, where $I$ denotes the identity map $I(x)=x$ on $A$, then $\varphi$ is irreducible. This is proved as follows. Suppose that $p \in A$ is a nonzero projection and that $\varphi(p) \leq \lambda p$, for some $\lambda \geq 0$. Then $(I+\varphi)^{k}(p) \leq(1+\lambda)^{k} p$, and so $1=R\left[(I+\varphi)^{k}(p)\right] \leq R\left[(1+\lambda)^{k} p\right]=p \leq 1$, implying that $p=1$.

Suppose that $A_{j}$ is a von Neumann subalgebra of $B(H)$, for $j=1, \ldots n$, and consider the operator algebra

$$
A=A_{1} \oplus A_{2} \oplus \cdots \oplus A_{n} \subset B(H \oplus \cdots \oplus H) .
$$

Let $\mathfrak{G}$ be a strongly connected graph on $n$ vertices and suppose that whenever there is a directed edge from vertex $i$ to vertex $j$ in the graph $\mathfrak{G}$, we have a specified positive linear map $\varphi_{i j}: A_{j} \rightarrow A_{i}$ such that $\varphi_{i j}(x)$ has dense range for every nonzero positive $x \in A_{j}$. Now "fill out" the matrix $\varphi=\left(\varphi_{i j}\right)_{1 \leq i, j \leq n}$ by arbitrarily choosing a positive map $\varphi_{\mu \nu}: A_{\nu} \rightarrow A_{\mu}$ whenever there fails to be a directed edge from vertex $\mu$ to vertex $\nu$ in the graph $\mathfrak{G}$. Standard matrix arguments (left to the reader) show that the positive linear map $\varphi$ on $A$ has the property that $(I+\varphi)^{n-1}$ is dense-range positive; by the remarks above on dense-range positivity, this conclusion implies that $\varphi$ is irreducible.

The spectral theory of Evans and Høegh-Krohn concerns operator algebras acting on finite-dimensional Hilbert spaces; it would be interesting, nevertheless, to have more complete information, beyond Corollary 1, Theorem 2, and Example 1, regarding the irreducibility of positive linear maps on infinite-dimensional operator algebras. For example, if one looks for an extension of Example 3, say, then the following statement, which is only a partial extension, is not difficult to prove: A completely positive map $\varphi$ on $A$ that generates an extremal ray in the cone of completely positive maps $A \rightarrow B(H)$ cannot be dense-range positive. The argument runs as follows. Suppose that $\varphi$ generates an extremal ray of the cone of all completely positive maps $A \rightarrow B(H)$. By [3, Cor1.4.3], there is an irreducible *-representation $\pi$ of $A$ on a Hilbert space $H_{\pi}$, and a nonzero operator $w: H \rightarrow H_{\pi}$ such that $w(H)$ is a cyclic subspace for $\pi(A)$ and $\varphi(x)=w^{*} \pi(x) w$ for every $x \in A$. Let $\xi \in H$ be a fixed unit vector for which $w \xi \neq 0$. Because $\pi$ is irreducible, by Kadison's transitivity theorem there is a nonzero $x \in A$ with $\pi(x) w \xi=0$; hence, $w \xi$ is in the kernel of $\pi\left(x^{*} x\right)$ and $\varphi\left(x^{*} x\right)$ has $\xi$ in its kernel, implying that the range of $\varphi\left(x^{*} x\right)$ fails to be dense in $H$. 


\section{REFERENCES}

1. C.A. Akemann and G.K. Pedersen, Facial structure in operator algebra theory, Proc. London Math. Soc. 64 (1992), 418-448. MR 93c:46106

2. S. Albeverio and R. Høegh-Krohn, Frobenius theory for positive maps on von Neumann algebras, Comm. Math. Phys. 64 (1978/79), 83-94. MR 81m:46091

3. W.B. Arveson, Subalgebras of $C^{*}$-algebras, Acta Math. 123 (1969), 141-224. MR 40:6274

4. M.-D. Choi, Completely positive linear maps on complex matrices, Linear Algebra Appl. 10 (1975), 285-290. MR 51:12901

5. M.-D. Choi and T.-Y. Lam, Extremal positive semidefinite forms, Math. Ann. 231 (1977), 1-18. MR 58:16512

6. D. Evans and R. Høegh-Krohn, Spectral properties of positive maps on $C^{*}$-algebras, J. London Math. Soc. 17 (1978), 345-355. MR 58:2319

7. F.R. Gantmacher, The theory of matrices, volume II, Chelsea, New York, 1959. MR 21:6372c

8. U. Groh, The peripheral point spectrum of Schwarz operators on $C^{*}$-algebras, Math. Z. 176 (1981), 311-318. MR 82i:46088

9. U. Groh, Some observations on the spectra of positive operators on finite-dimensional $C^{*}$ algebras, Linear Algebra Appl. 42 (1982), 213-222. MR 84f: 46079

10. S.-H. Kye, Facial structures for positive linear maps between matrix algebras, Canad. Math. Bull. 39 (1996), 74-82.

11. M. Mathieu, Elementary operators on prime $C^{*}$-algebras, I., Math. Ann. 284 (1989), 223-244.

12. H.H. Schaefar, Banach Lattices and Positive Operators, Springer, New York, 1974. MR 54:11023

13. H. Schneider, Positive operators and an inertia theorem, Numer. Math. 7 (1965), 11-17. MR 30:3888

14. E. Stormer, Cones of positive maps, Contemporary Math. 62 (1987), 345-356. MR 87m:46111

Department of Mathematics and Statistics, University of Regina, Regina, SaskatChewan, Canada S4S 0A2

E-mail address: farenick@abel.math.uregina.ca 